\title{
An Effective Antenna Array Diagnosis Method via Multivalued Neural Network Inverse Modeling Approach
}

\section{Oluwole John Famoriji, and Thokozani Shongwe}

Department of Electrical and Electronic Engineering Science, University of Johannesburg, P.O. Box 524, Auckland Park, 2006, Johannesburg, South Africa.

Corresponding author: Oluwole J. Famoriji (e-mail: famoriji@mail.ustc.edu.cn).

\begin{abstract}
Failure of element (s) in antenna arrays impair (s) symmetry and lead to unwanted distorted radiation pattern. The replacement of defective elements in aircraft antennas is a solution to the problem, but it remains a critical problem in space stations. In this paper, an antenna array diagnosis technique based on multivalued neural network ( $\mathrm{mNN}$ ) inverse modeling is proposed. Since inverse analytical input-to-output formulation is generally a challenging and important task in solving the inverse problem of array diagnosis, ANN is a compelling alternative, because it is trainable and learns from data in inverse modelling. The $\mathrm{mNN}$ technique proposed is an inverse modelling technique, which accommodates measurements for output model. This network takes radiation pattern samples with faults and matches it to the corresponding position or location of the faulty elements in that antenna array. In addition, we develop a new training error function, which focuses on the matching of each training sample by a value of our proposed inverse model, while the remaining values are free, and trained to match distorted radiation patterns. Thereby, $\mathrm{mNN}$ learns all training data by redirecting the faulty elements patterns into various values of the inverse model. Therefore, $\mathrm{mNN}$ is able to perform accurate array diagnosis in an automated and simpler manner. We demonstrate the proposed technique in proof-of-concept via an HFSS-based simulation of $10 \times 10$-array of waveguides in realistic noise scenario. The numerical results confirm the appropriateness and effectiveness of the proposed method.
\end{abstract}

INDEX TERMS Antenna measurement, array diagnosis, mNN, fault detection, electromagnetic sensing, and inverse modeling.

\section{INTRODUCTION}

F ailures identification and detection in large antenna array is a relevant topic both in theory and in practice with various applications in both military and civilian market. Current and future technologies employ sophisticated active or phased arrays with large elements. For example, large array employed in RADAR systems, full MIMO systems, massive MIMO, and personal communication devices that require complex antenna arrays. As a result, there will always be a demand for fast and accurate complex antenna systems diagnosis, to resolve the unacceptable radiation pattern distortion caused by element (s) failure in the array (Figure.1).

Many antenna array diagnosis methods, based on genetic algorithms [1], [2], exhaustive search [3], matrix inversion [4], and MUSIC [5], have been developed in literature to identify faulty antenna elements in an array. All the methods compare the array under test (AUT) with the radiation pattern of an "error free" reference array. All the methods in [1-5] need big measurement samples for large antenna arrays, to get reliable diagnosis. Reducing the measurement samples, compressive sensing (CS) based techniques have been reported in [6]-[9]. Despite the compelling outcome, the methods in [1]-[9] focused on the detection of sparsity pattern of a failed array, i.e. failed elements location, not on the complex blockage, as addressed in [10]. Moreover, CS methods exhibit various limitations, such as: (a) they required taking measurements in various receive locations, and they are not appropriate when both receiver and transmitter are fixed. (b) They assume "error free" receive antennas (i.e. only fault at AUT), which is not always true in reality because fault can occur at transmitter and receiver. (c) they do not perform optimization on CS measurement matrices. The shortcomings of CS based methods [6][10], and the high time of diagnosis required by methods in [1]-[5] and complexity in measurement time cost motivate the need for an intelligent antenna array diagnosis method. Inspired and motivated by [11], an approach based on artificial neural network is presented to model the inverse radiation problem for automated and simple antenna array diagnosis.

ANNs are identified to be a useful and powerful microwave imaging and design tool [12], [13]. This method has been deployed in various areas such as microwave components parametric modeling [14], [15], and modeling of microwave devices [16], [17]. $\mathrm{ANN}$ is capable of learning the existing non-linear relationships 


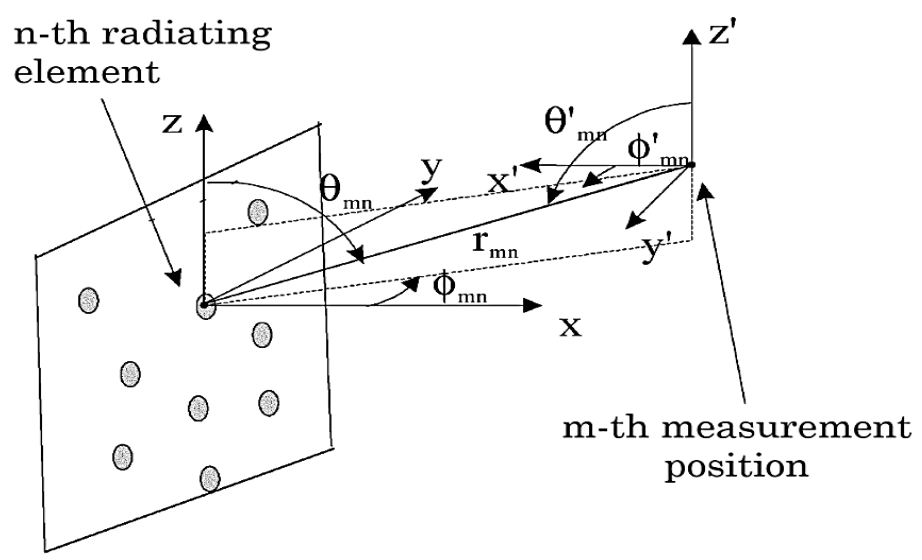

FIGURE 1. Geometry problem of array diagnosis.

between antenna element features and the radiation patterns. The ANN trained to model the antenna under test (AUT) is a forward model, with radiation patterns as inputs to the model, and elements parameters (e.g. position and location) as the output. For the purpose of array diagnosis, the radiation patterns are processed in reverse direction so as to detect the location of faults from based on the reference radiation patterns (a priori knowledge), which is referred to as inverse problem. Two approaches are usually employed to in solving inverse problem: the most conventional optimization approach, and inverse modeling approach. For optimization approach, the forward models is iteratively evaluated to detect the faulty elements, such that the AUT matches the reference array (RA). The optimization stage is to detect the faults, which is usually repeated over and over again, thereby consumes excessive.

Inverse modeling method is the second approach to solving inverse problem [16]. The electrical parameters (radiation patterns, in this case) are given as inputs, while physical or geometrical information (elements position) are given as the corresponding outputs of the model. After the inverse model is obtained, it immediately provides the physical or geometrical parameters (faulty elements) with no need of evaluating the model iteratively for particular radiation patterns. This approach is faster than the traditional iterative process of optimization. However, the inverse model analytical formula or function is not easy to get. Thereby, ANN has been employed to learn the input-and-output relationship in inverse problem [18], [19].

Despite the high speed exhibited by inverse model, training the model very well is not an easy task in array diagnosis. This is because of the non-uniqueness problem that exist in the input-tooutput relationship. Non-uniqueness problem implies the different training samples with identical inputs having contradictory or conflicting output parameters. Another difficult issue is the various radiation patterns with comparable but non-identical input parameters having very wide gap difference in output values. This consequently causes ambiguity in both contradictory and non-contradictory samples. As a result of impossibility in training the $\mathrm{NN}$ to simultaneously map conflicting/contradictory parameters at the output for uniform input samples, the error in training and the accuracy of the model become large and low, respectively. This non- uniqueness problem has been addressed in [20]. The contradictory samples are grouped according to derivative data via the forward model of the $\mathrm{NN}$, so that each groups are with no contradictory or conflicting data problem. Multiple inverse models are developed depending on the divided data groups, and are then juxtaposed to formulate a full model.

This study aims at further simplification of the inverse modeling procedure and resolve the non-uniqueness problem in more automated and simpler manner for antenna array diagnosis. In low dimensional spaces, manual data division or segmentation and multiple sub-models training mitigate the non-uniqueness problem, while in high dimensional spaces, non-uniqueness problem becomes more complex as inputs and outputs increases. Manually segmented data does not lead to easy removal of the non-uniqueness issue. In addition, there are some difficult cases where conflicting data in short distance is higher than what the derivative information will differentiate. Furthermore, contradictory data can be of identical derivative symbols. In such situation, the symbol derivative information is insufficient to differentiate multiple contradictions, even after exhaustively making use of derivative information. Therefore, to solve the problem of non-uniqueness, a more systematic approach is needed.

This work employs a multivalued neural network (mNN)based inverse modeling method, which associates a group of radiation patterns with multiple group of physical or geometrical elements parameter for array diagnosis purpose. A physical or geometrical set of elements parameters is referred to as a value in our developed model. This developed $\mathrm{mNN}$ is designed to adapt multiple values for the model output. In addition, a training error function is proposed towards each training sample matching with single value of the developed inverse model, and other values are trained to match other contradictory samples. As such, our proposed neural network (NN) learns all training data via automatic redirection of the conflicting information into various values of our inverse model. Hence, the developed method resolves the non-uniqueness issue in a more automated and easier manner compared to previous $\mathrm{NN}$ inverse methods. Inverse modeling for the diagnosis of a $10 \times 10$ antenna array configuration illustrations with failures demonstrate our method. This method decreases measurements sample remarkably, 
making us to treat various important concerns such as energy consumption, processing complexity, and speed. The innovations and main contributions of this paper are summarized as follows:

I. We present an optimal antenna array diagnosis method by means of multivalued neural network. The proposed method is able to solve the associated non-uniqueness problem (i.e. the problem of different training samples with identical inputs having contradictory outputs). It has lower training error and test error compared to the conventional direct inverse model.

II. We proposed a training procedure and test error functions towards optimal antenna array diagnosis. They are tested with various electromagnetic radiation pattern acquired from a designed antenna array.

III. Our low latency multivalued NN-based method provides accurate diagnosis of array in a simpler and automated way with reduced energy consumption and processing complexity.

\section{ANTENNA ARRAY DIAGNOSIS PROBLEM}

Antenna array diagnosis is considered a forward problem from physical or geometrical parameters to electrical parameters of the elements. Assuming $m$ and $n$ represent the inputs and outputs of the forward problem, respectively. Let $\mathbf{y}$ be m-vector with input information of the forward problem, which are the geometrical locations of elements in the array. Also, let $\boldsymbol{w}$ be $n$-vector that contains the output information, which are the antennas radiation patterns. Hence, the forward problem is defined as

$$
\boldsymbol{w}=\boldsymbol{f}(\mathbf{y})
$$

$\boldsymbol{f}$ is the relationship between the input and output of the forward problem.

To ensure adequate antenna array diagnosis, we process the information in reverse direction in order to identify the location of faults from a specific radiation patterns. This is the inverse problem in which both the inputs and the outputs are the inversion of those in the forward problem.

\section{DIRECT INVERSE MODEL}

One of the technique available for solving inverse problem is inverse modeling. The inputs to the inverse model are the radiation patterns while the outputs are the location or position of the elements. Due to non-availability of mathematical formula for inverse input-output relationship, ANN thereby logically becomes an alternative because it is trainable and learns from inverse modeling data. ANN is trained by data gotten by swapping the input-output data from the forward problem in the direct inverse modeling approach [21]. Here, $n$ and $m$ denote number of inputs and outputs of inverse model of the array. Assuming $\mathbf{x}$ is an $n$-vector that contains the inputs to the inverse model, which are the radiation patterns. $\boldsymbol{y}$ is equally assumed to be the outputs of the inverse model, which are the location parameters of the elements. Hence, the direct NN inverse model is

$$
y(\mathbf{x}, \varphi)=f^{-1}(\mathbf{x})
$$

as such $\boldsymbol{f}^{-\mathbf{1}}$ is the relationship that exist between the input and output of the inverse problem, $\boldsymbol{y}(\mathbf{x}, \boldsymbol{\varphi})$ denotes the $\mathrm{NN}$ with $\mathbf{x}$ as inputs and $\boldsymbol{y}$ as the outputs, and $\boldsymbol{\varphi}$ as the vector that contains the NN weights. The $\varphi$ vector elements contains the bias values for entire neuron in each layer, and the weights values that links the neurons in each layers.

Direct inverse model is usually obtained via the process of training. The $i$ th pair of training sample is $\left(\mathbf{x}_{i}, \mathbf{b}_{i}\right), i \in \boldsymbol{P}_{r}$, where subscript $i$ defines the index of sample and $\boldsymbol{P}_{r}$ denotes index set of the entire training samples. $\mathbf{x}_{i}$ defines the $i$ th samples of $\mathbf{x} . \mathbf{b}_{i}$ is the $i$ th sample of $\boldsymbol{y}$ which corresponds to input $\mathbf{b}_{i}$. The traditional training error formula of the direct inverse modeling problem is given as

$$
\boldsymbol{E}(\boldsymbol{\varphi})=\sum_{i \in \boldsymbol{P}_{r}} \frac{1}{2}\left\|\boldsymbol{y}\left(\mathbf{x}_{\boldsymbol{i}}, \boldsymbol{\varphi}\right)-\boldsymbol{b}_{i}\right\|^{2}
$$

$\boldsymbol{y}\left(\mathbf{x}_{i}, \boldsymbol{\varphi}\right)$ is the output of NN for the input $\mathbf{x}_{i}$ [22].

For relatively easy inverse problem, for instance, the relationship between the input and the output is unique; here the model accuracy is high as the training is easy. In antenna array, there is an inverse input-to-output non-uniqueness problem in the relationship of training data (radiation pattern). A training error becomes larger as the model cannot be adequately trained.

Due to the impossibility of training an ANN model to simultaneously match many conflicting outputs values for the same input values, as such, the training error in Eqn. (3) remains large. Manual data division/segmentation is unable to easily solve the nonuniqueness problem [20], [23]. Hence, an approach that solves the non-uniqueness problem is very important for proper antenna array diagnosis.

\section{PROPOSED MULTIVALUED NN INVERSE MODEL}

Here, we develop a mNN inverse modeling approach to successfully associate a set of radiation patterns with multiple sets of array elements location/position. A set of position in the array is termed one element of our proposed inverse model. The multivalued $\mathrm{NN}$ is designed to contain multiple elements for output of the model. Against manual detection of contradictory data in training set, we use NN learning capability to redirect conflicting or contradictory information automatically into different values of the model proposed. We denote $N$ to be the number of elements of our developed inverse model. Therefore, we proposed $\mathrm{mNN}$ model by repetition of the direct inverse model output in Eqn. (2) $N$ times to become

$$
\boldsymbol{z}_{A N N}(\mathbf{x}, \boldsymbol{\varphi})=\left[\begin{array}{lllll}
\boldsymbol{y}_{1}^{T} & \boldsymbol{y}_{2}^{T} & \boldsymbol{y}_{3}^{T} & \boldsymbol{y}_{4}^{T} \ldots & \boldsymbol{y}_{N}^{T}
\end{array}\right]
$$

where $\mathbf{z}_{A N N}$ is the MLP (multilayer perceptron) that represents the developed mNN inverse model. $\boldsymbol{y}_{k}$ denotes $k$ th value of our inverse model for input $\mathbf{x}, k=1,2,3, \ldots, N$. Individual element $\boldsymbol{y}_{k}$ of the 


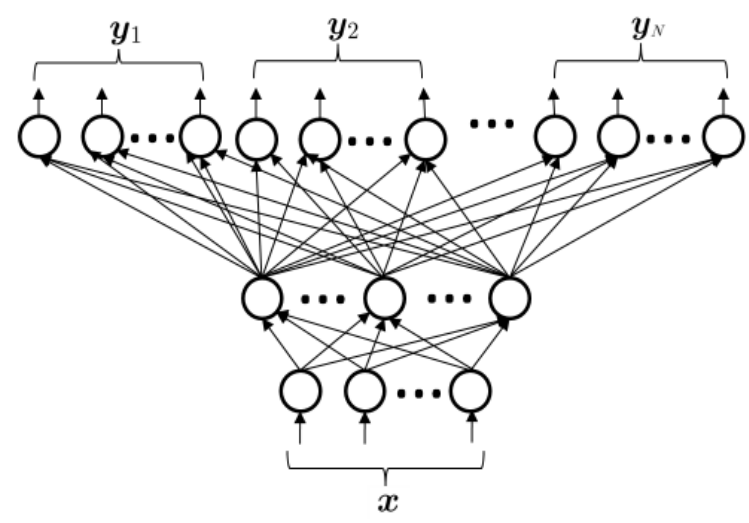

FIGURE 2. mNN inverse modeling towards accurate and efficient antenna array diagnosis.

developed $\mathrm{mNN}$ is an $m$-vector that contains geometry of each element defined in a direct way of inverse modeling technique. Figure 2 shows a typical example of our proposed method.

For easy representation of $\boldsymbol{y}_{k}$ via the proposed $\boldsymbol{z}_{A N N}$, we can define a $m \times(N \cdot m)$ matrix $\boldsymbol{S}_{k}$ which contains zeros every position except for $(h,(k-1) \cdot m+h)^{t h}$ position, where the element is 1 , for $h=1,2,3, \ldots, m$. Thereby, $\boldsymbol{y}_{k}$ can be computed from

$$
\boldsymbol{y}_{\boldsymbol{k}}=\boldsymbol{S}_{k} \cdot \mathbf{z}_{A N N}(\mathbf{x}, \boldsymbol{\varphi})
$$

$$
k=1,2,3, \ldots, N \text {. }
$$

Due to the increase in the total outputs number of $\boldsymbol{z}_{A N N}$ to $N \cdot m$, the formula for training error in Eqn. (3) becomes unsuitable for $\mathrm{mNN}$. Therefore, the brute force technique needed to resolve the training issue of $\mathrm{mNN}$ is to re-format all training data, where individual training sample containing all conflicting outputs for the same inputs. Conversely, for contradictories, in which input values are incompletely the same but very much alike, while the output values completely vary, the conflicting data are unable to be jointly reformatted. Data re-formatting technique fails in the training process of our inverse model. Thus, a new training error formula for the $\mathrm{mNN}$ $\mathbf{z}_{A N N}$ is proposed, such that $\mathrm{mNN}$ can redirect conflicting and/or contradictory samples into other values of our developed inverse model, and learn the entire training data ignoring the complexity of detecting and sorting-out of the whole multivalued solutions.

\section{V.TRAINING PROCEDURE AND TEST ERROR}

\section{FORMULAS OF THE DEVELOPED MNN INVERSE MODEL}

Making sure that the developed method is capable of learning contradictory and conflicting data within the training set, in a way that different elements of $\boldsymbol{y}_{k}$, for $\mathrm{k}=1,2,3, \ldots, \mathrm{N}$, able to map various contradictories for very similar or the same input $\mathrm{x}$, therefore we present a new training error to be

$$
\boldsymbol{E}(\varphi)=\sum_{i \in P_{r}} E_{i}(\varphi)
$$

$P_{r}$ denotes the index set of all the training samples and $\boldsymbol{E}_{\boldsymbol{i}}(\boldsymbol{\varphi})$ represents the training error for the ith training sample defined as

$$
\boldsymbol{E}_{\boldsymbol{i}}(\boldsymbol{\varphi})=\left(\sum_{k=1}^{N} \frac{1}{e_{k, i}(\boldsymbol{\varphi})}\right)^{-1}
$$

$e_{k, i}(\boldsymbol{\varphi})$ represents the error between $\boldsymbol{y}_{k}$ and $\boldsymbol{b}_{i}$ as

$$
\begin{aligned}
e_{k, i}(\boldsymbol{\varphi})=\frac{1}{2} \| \boldsymbol{y}_{k}- & \boldsymbol{b}_{i} \|^{2} \\
& =\frac{1}{2} \| \boldsymbol{S}_{k} \cdot \boldsymbol{z}_{A N N}\left(\mathbf{x}_{i}, \boldsymbol{\varphi}\right) \\
& -\boldsymbol{b}_{i} \|^{2}
\end{aligned}
$$

$$
K=1,2,3, \ldots, N
$$

$\boldsymbol{y}_{k}$ represents the kth value of our developed inverse model $\boldsymbol{z}_{A N N}\left(\mathbf{x}_{i}, \boldsymbol{\varphi}\right)$ for input $\mathbf{x}_{i}$. If an element $\boldsymbol{y}_{k}$ of our developed model fits the training sample $\boldsymbol{b}_{i}$ correctly, this implies that the error $e_{k, i}(\boldsymbol{\varphi})$ of this particular element is infinitesimal. The infinitesimal error $e_{k, i}(\boldsymbol{\varphi})$ of an element results in an infinitesimal value in the developed total error function $\boldsymbol{E}_{\boldsymbol{i}}(\boldsymbol{\varphi})$ of Eqn. (7) for the ith sample. Concurrently, the $e_{k, i}(\varphi)$ for all other elements are large, implying the remaining elements are free and maybe different from the present training sample $\boldsymbol{b}_{i}$. Hence, the developed error formula $\boldsymbol{E}_{\boldsymbol{i}}(\boldsymbol{\varphi})$ of Eqn. (7) tailored towards matching of the training sample with one of the elements of $\mathrm{mNN}$ model that is the nearest to the present training sample and neglecting other elements.

The ANN weights $\boldsymbol{\varphi}$ are initially conditioned to be small numbers [23], at the start of the training process. We applied quasiNewton optimization method to reduce training error presented in Eqn. (6) where $\boldsymbol{\varphi}$ are the variables of optimization. ANN weights $\boldsymbol{\varphi}$ are updated during training process by optimization.

After the successful training, the inverse model test is performed via test data. Due to the fact that our proposed inverse model is designed to handle multiple parameters or values for the output of the model, the traditional function presented in Eqn. (3) is unsuitable for the model. Hence, we present a test error formula appropriate for the developed $\mathrm{mNN}$ inverse model is

$$
\begin{array}{r}
\boldsymbol{P}(\boldsymbol{\varphi})=\sum_{i \in P_{v}} \min _{k}\left\{\frac{1}{2}\left\|\boldsymbol{y}_{k}-\boldsymbol{b}_{i}\right\|^{2}\right\} \\
=\sum_{i \in P_{v}} \min _{k}\left\{\frac{1}{2} \| \boldsymbol{S}_{k} \cdot \boldsymbol{z}_{A N N}\left(\mathbf{x}_{i}, \boldsymbol{\varphi}\right)\right. \\
\left.-\boldsymbol{b}_{i} \|^{2}\right\} \\
\mathrm{k}=1,2,3, \ldots, \mathrm{N}
\end{array}
$$

$P_{v}$ denotes the index set for all the test samples. Using the developed test error formula in Eqn. (9), we compute the error for all the elements of the proposed inverse model, and of cause, the nearest element to the test data was selected for the total error test computation. In addition, the error formula generates a metrics to test error compatibility to the traditional NN of Eqn. (3). 


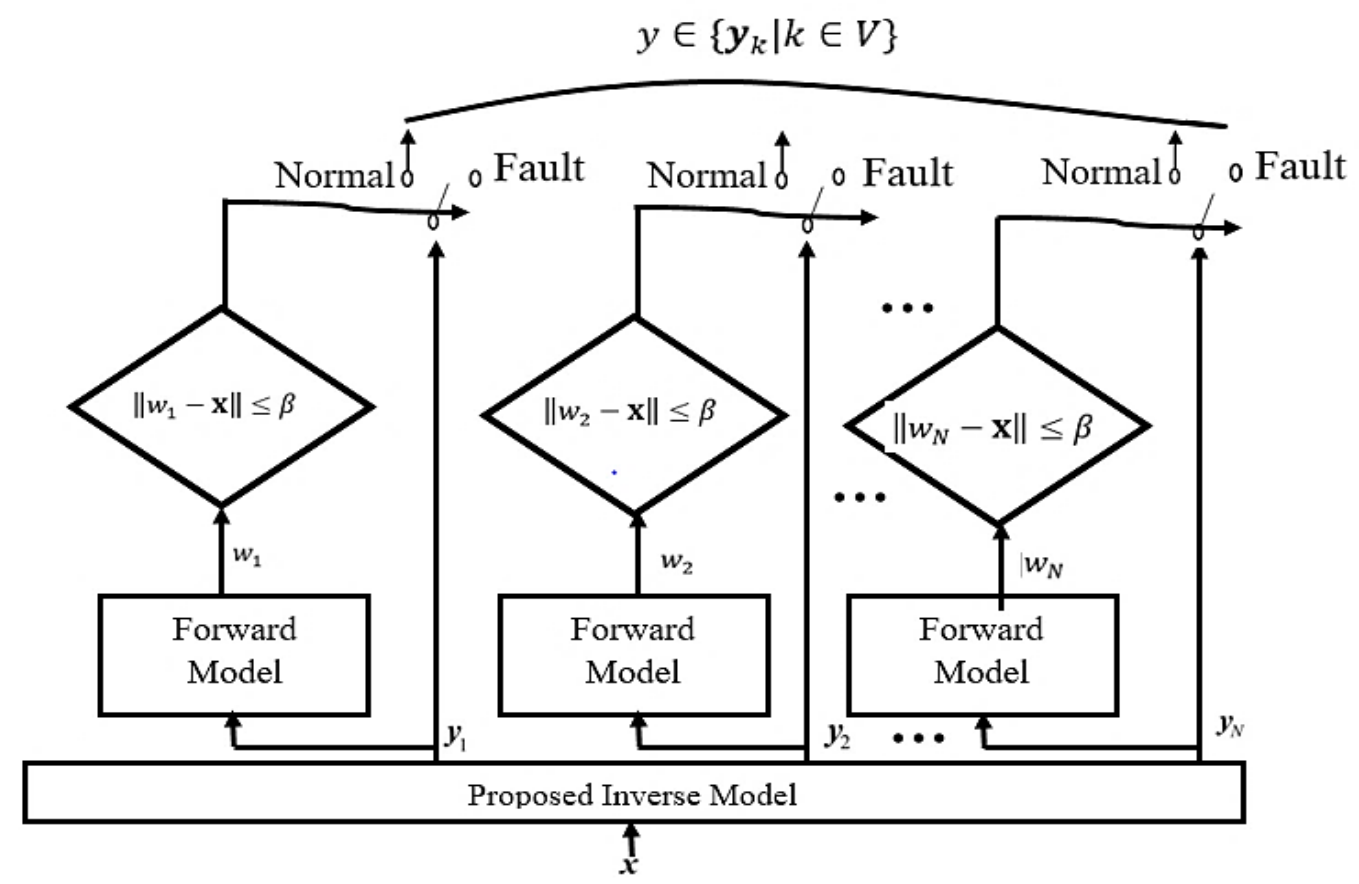

FIGURE 3. Fault detection technique of the proposed multivalued NN inverse model.

\section{PROPOSED FAULT DETECTION IN ANTENNA ARRAYS}

To ensure accurate inverse model, the number of elements in the array $N$ of $\mathrm{mNN}$ should be the same as the total number of possible faults within training data for all inputs. Conversely, following the successful NN training using the error functions: Eqns. (6), (7), and (8), the number of faults which is usually not the same as $N$ for a specific input in practice. Therefore, all $\mathrm{NN}$ inverse model values are accurate. The number of possible faults are lower than $N$. In this case, the $N$ elements given by our developed inverse model indicates some elements as normal while some as faulty. Since $\mathrm{mNN}$ always give $N$ output values of the model for a specific input, there is a need to come up with a technique to differentiate normal elements from the faulty elements in the entire elements $\boldsymbol{y}_{k}$ for $k=1,2,3, \ldots, N$.

Here, we use forward model to decide the normal elements. Each $\boldsymbol{y}_{k}$ from our inverse model acts as inputs to the forward model in Eqn. (1), $k=1,2,3, \ldots, N$. At the end of the computation of forward model, then we obtain $N$ output. $w_{k}$ defines the forward model output for $\boldsymbol{y}_{k}, k=1,2,3, \ldots, N$. The forward model output $w$ and the inverse model input $\mathbf{x}$ exhibit the same parameters. Apparently, the errors $\boldsymbol{E}_{0}$ that exist between $w_{k}$ and $\mathbf{x}$ for various $\boldsymbol{y}_{k}$ informs us which elements are faulty and which elements are normal in the array. $\boldsymbol{E}_{0}\left(\boldsymbol{y}_{k}\right)$ can then be expressed as

$$
\boldsymbol{E}_{0}\left(\boldsymbol{y}_{k}\right)=\left\|\boldsymbol{w}_{\boldsymbol{k}}-\mathbf{x}\right\|
$$

where $\boldsymbol{w}_{\boldsymbol{k}}=\boldsymbol{f}\left(\boldsymbol{y}_{k}\right), k=1,2,3, \ldots, N$ and $\boldsymbol{f}(\cdot)$ defines our forward model in Eqn. (1), $\boldsymbol{y}_{k}$ as input, and $\boldsymbol{w}_{\boldsymbol{k}}$ as output.
If

$$
\boldsymbol{E}_{0}\left(\boldsymbol{y}_{k}\right)=\left\|\boldsymbol{f}\left(\boldsymbol{y}_{k}\right)-\mathbf{x}\right\| \leq \beta
$$

is satisfied, $\boldsymbol{y}_{k}$ is the normal element. $\beta$ is a constant predefined here.

If

$$
\boldsymbol{E}_{0}\left(\boldsymbol{y}_{k}\right)=\left\|\boldsymbol{f}\left(\boldsymbol{y}_{k}\right)-\mathbf{x}\right\|>\beta
$$

$\boldsymbol{y}_{k}$ is referred to as the faulty element.

Let index set of valid values be denoted as $V$, then

$$
V=\left\{k \mid \boldsymbol{E}_{0}\left(\boldsymbol{y}_{k}\right) \leq \beta ; k=1,2,3, \ldots, N\right\}
$$

Hence, the normal elements for $\mathrm{mNN}$ inverse model is given as

$$
y \in\left\{\boldsymbol{y}_{k} \mid k \in V\right\}
$$

The detection of faulty and normal elements of the developed inverse model is diagrammatically demonstrated in Figure 3. The statistical measures calculated herein for the evaluation of the multivalued ANN performance are the reconstruction error expressed as

$$
\xi=100 \times \frac{\sum_{k=1}^{K}\left|y_{k}-\hat{y}_{k}\right|^{2}}{\sum_{k=1}^{K}\left|y_{k}\right|^{2}}
$$




$$
M=\left[\begin{array}{cccccccccc}
m_{11} & m_{12} & m_{13} & m_{14} & m_{15} & m_{16} & m_{17} & m_{18} & m_{19} & m_{1,10} \\
m_{21} & m_{22} & m_{23} & m_{24} & m_{25} & m_{26} & m_{27} & m_{28} & m_{29} & m_{2,10} \\
m_{31} & m_{32} & m_{33} & m_{34} & m_{35} & m_{36} & m_{37} & m_{38} & m_{39} & m_{3,10} \\
m_{41} & m_{42} & m_{43} & m_{44} & m_{45} & m_{46} & m_{47} & m_{48} & m_{49} & m_{4,10} \\
m_{51} & m_{52} & m_{53} & m_{54} & m_{55} & m_{56} & m_{57} & m_{58} & m_{59} & m_{5,10} \\
m_{61} & m_{62} & m_{63} & m_{64} & m_{65} & m_{66} & m_{67} & m_{68} & m_{69} & m_{6,10} \\
m_{71} & m_{72} & m_{73} & m_{74} & m_{75} & m_{76} & m_{77} & m_{78} & m_{79} & m_{7,10} \\
m_{81} & m_{82} & m_{83} & m_{84} & m_{85} & m_{86} & m_{87} & m_{88} & m_{89} & m_{8,10} \\
m_{91} & m_{92} & m_{93} & m_{94} & m_{95} & m_{96} & m_{97} & m_{98} & m_{99} & m_{9,10} \\
m_{10,1} & m_{10,2} & m_{10,3} & m_{10,4} & m_{10,5} & m_{10,6} & m_{10,7} & m_{10,8} & m_{10,9} & m_{10,10}
\end{array}\right]
$$

where $y_{k}$ and $\hat{y}_{k}$ are the real and predicted failure of $\mathbf{y}$, respectively. Given this relationship, performance analysis is easily conducted Figure 4 show the reconstruction error versus SNR for different number of failures $V_{f}$. The reconstruction error $\xi$ is high when SNR is low for any number of failure $V_{f}$, of failures $V_{f}$. The reconstruction error $\xi$ is high when SNR is low for any number of failure $V_{f}$, while there is better performance at higher SNR for all number of failure. We also tested the effect of failure rate on the performance of the technique by plotting reconstruction error against the number of failures in Figure 5. As expected, the performance decreases with higher number of failed elements. Figures 6 and 7 show the plot of reconstruction error against the measurements for different SNR and failure, respectively. Reconstruction error decreases when there is increase in the measurements. Low SNR leads to higher error, and low failure leads to low error. All the results show how reasonable our method is, for antenna array diagnosis.

\section{VII.NUMERICAL EXPERIMENT, RESULTS AND DISCUSSION}

The radiated field of the array with $N$ elements can be expressed as:

$$
\vec{E}=\sum_{n=1}^{N} a_{n} \vec{E}_{n}=\sum_{n=1}^{N} q_{n} e^{-j \phi_{n}} \vec{E}_{n}
$$

good condition is depicted in Fig. 9. In this work, the maximum number of faults considered is where $\vec{E}_{n}$ denoted the normalized field of the $n$th element and $a_{n}$ is the corresponding complex excitation with amplitude $x_{n}$ and phase $\phi_{n}$, respectively. The change in amplitude $\Delta q_{n}=q_{n}^{R e f}-q_{n}^{A U T}$ is the amplitude of the $n$th element. We made a hypothesis that few numbers of elements are faulty. This is usually true in practice because AUT diagnosis should be conducted when AUT performance is degraded. Only two element excitation states are considered (i.e. normal operation and total failure).

Failure of element (s) in antenna array causes field intensity variation across the aperture of the array, leading to increase in ripple level and sidelobe of the radiation pattern. From the perspective of faulty element in antenna array, it is observed that the size and number of sidelobes and generally the power pattern depends on the position and number of faulty elements in array. It is equally important to note that faulty elements have effect on the total array radiation pattern. Increase in number of faults causes increase variation in radiation pattern. The task here is to generate a mapping between distorted radiation pattern and corresponding element position in the array. The proposed MLP performs the task. The mNN inverse model takes in radiation patterns as input and provides the position of faulty elements as output.

In this paper, we demonstrate the effectiveness of our proposed inverse modeling method in the absence of experimental facility. A real failed element in antenna array is not performed, but simulated. We consider 100 elements made up of an open-ended waveguide $10 \times 10 \mathrm{WR} 90$ array operating at $27 \mathrm{GHz}$ as shown in Figure 8 , and the simulated radiation pattern with no element failure at $\phi=0^{0}$ principal plane. The array is of $24.88 \times 12.18 \mathrm{~mm}^{2}$ aperture size and is uniformly spaced in both $x$ and $y$ directions by $\lambda$ and $\lambda / 2$, respectively. The popular full wave EM software Ansys HFSS v.19 was used to compute the radiation pattern of the array. Here, it may be stated that the application of ANN in this work includes the mutual coupling effect.

At first, all the elements $N$ are equally excited to emulate reference array (i.e. array without failure). After, $V$ number of random failures in the amplitude excitation are initiated in order to emulate and model the AUT, and the corresponding radiation patterns were evaluated in the same $\phi=0^{0}$ principal plane, as all the elements in good condition. The radiation pattern in $\phi=0^{0}$ principal plane with all elements in five. The same technique can be extended to higher number of faults. However, in real situation, the probability of such high number of failure is very low. Also in practice, measurements are usually corrupted by noise; hence, a Gaussian noise is added to the patterns, such that the noise level is decided by signal-to-noise ratio (SNR). The SNR is estimated from maximum magnitude of the received signal fit with dynamic measurement range. Therefore, noise can be expressed as

$$
\boldsymbol{n}=\frac{\mathbb{N}(0,1)+j \mathbb{N}(0,1)}{\sqrt{2}} \max |\boldsymbol{y}| \cdot 10^{-S N R_{d B} / 20}
$$

where $\mathbb{N}(0,1)$ denotes Gaussian random vector of 0 mean and 1 standard deviation.

\section{A) Data Generation}

We generated training data by simulation of radiation patterns for random location of non-radiating elements (i.e. failures) with one, two, three, four, and five element faults. Then the radiation patterns were sampled. The sampling of radiation pattern is justified, since it 

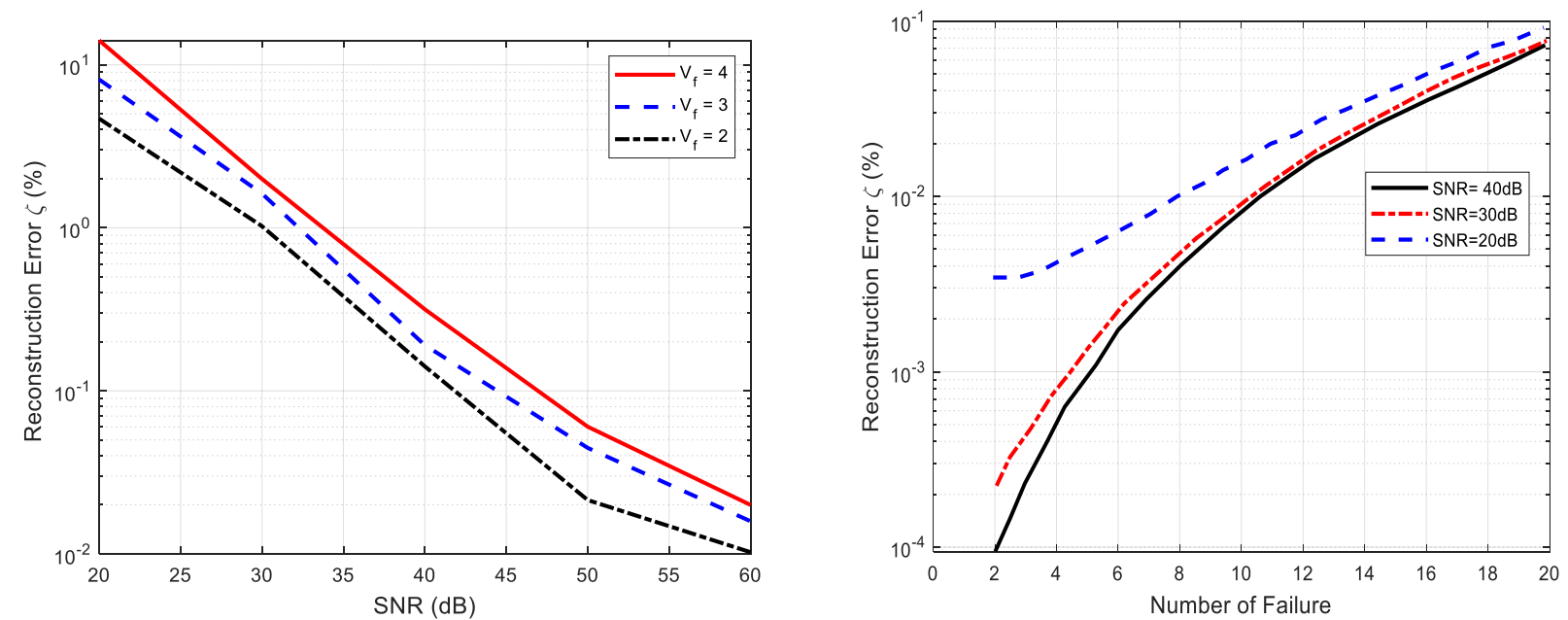

FIGURE 4. Reconstruction error versus SNR for different number of failure. FIGURE 5. Reconstruction error versus number of failure for different SNR.
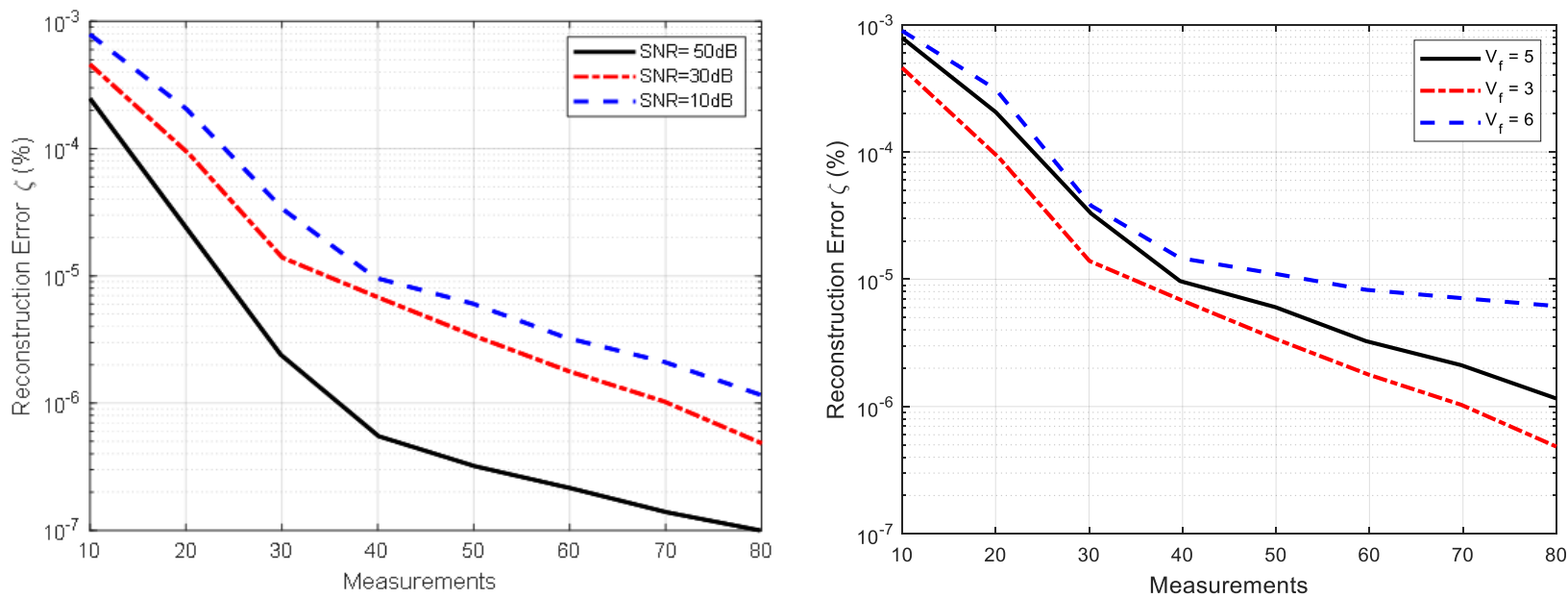

FIGURE 6. Reconstruction error versus measurements for different SNR. FIGURE 7. Reconstruction error versus measurements for different number of failure.

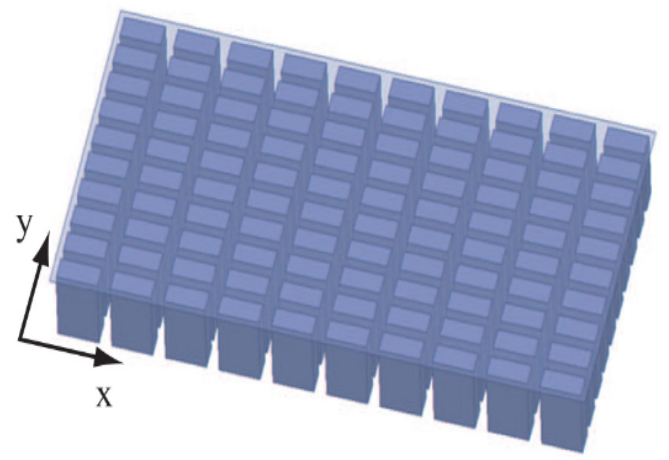

FIGURE 8. HFSS-based open ended $10 \times 10$ waveguide array for the diagnostic demonstration. 


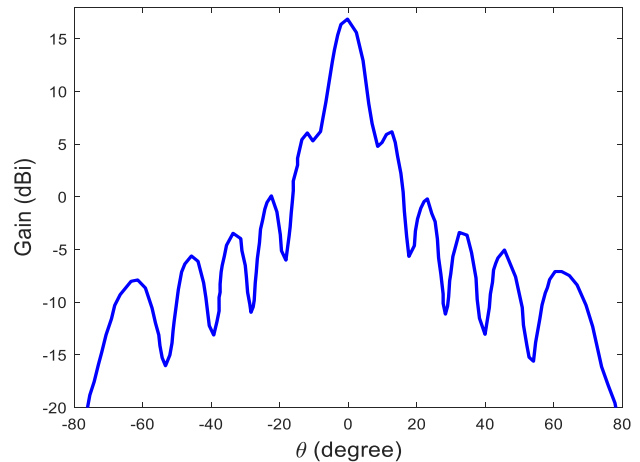

FIGURE 9. $10 \times 10$ array simulated radiation pattern with no element failure at $\phi=0^{0}$ principal plane.

TABle I. TRAining PARAMETERS

\begin{tabular}{|c|c|}
\hline Parameter & Value \\
\hline Number of input neurons & 46 \\
\hline Number of output neurons & 5 \\
\hline Number of hidden layers & 1 \\
\hline Number of hidden neurons & 17 \\
\hline Learning rate & 0.05 \\
\hline Training Time & 35 minutes \\
\hline
\end{tabular}

Table II. Modeling Results VIA Different InVERSE Modeling TeCHNIQUeS FOR $10 \times 10$ ANTENNA ARRAY

\begin{tabular}{|c|c|c|}
\hline Inverse Modeling Approach & $\begin{array}{c}\text { Training } \\
\text { Error }(\%)\end{array}$ & $\begin{array}{c}\text { Test Error } \\
(\%)\end{array}$ \\
\hline Direct inverse modeling approach & 6.01 & 5.82 \\
\hline mNN based inverse modeling & 3.34 & 3.98 \\
\hline mNN based inverse modeling with 2 faults & 2.79 & 3.13 \\
\hline mNN based inverse modeling with 3 faults & 2.42 & 2.76 \\
\hline mNN based inverse modeling with 4 faults & 2.18 & 2.41 \\
\hline mNN based inverse modeling with 5 faults & 1.88 & 2.05 \\
\hline
\end{tabular}

is impossible to get the whole distorted pattern at the base station for an antenna array in space. Because principal plane provide us sufficient information to obtain substantial results [25]-[27], samples were taken from the pattern in the principal plane $\phi=0^{0} .46$ samples were taken in the interval of 20 in xy and yz plane from each radiation pattern to formulate the input data set for training. The corresponding position of faulty element (s) is the output data. This implies the output data is a matrix M which consists of $m_{i j}$ elements. Different approaches of selecting output data to identify faults is possible and depends on the user. For the current consideration, the output matrix $\mathrm{M}$ is defined as Eqn. (18). HFSS and MATLAB softwares were used for data generation and implementation of the $\mathrm{mNN}$, respectively. The simulation is conducted with both softwares installed on personal HP PAVILION laptop computer (1-TB memory, 16G RAM, and Intel core i7-8565U CPU@1.80GHz 1.99 GHz).

\section{B) Training of Network}

Here, we trained the MLP (in conjunction with Eqn. (4)) in backpropagation mode, because of its capability for forming mapping implementations. According to the weight updation of backpropagation training algorithm is

$$
\boldsymbol{\varphi}_{k}^{i+1}=\boldsymbol{\varphi}_{k}^{i}-\eta \frac{\partial \boldsymbol{E}_{0}\left(\boldsymbol{y}_{k}\right)}{\partial \boldsymbol{\varphi}_{k}}
$$

where $\boldsymbol{E}_{0}\left(\boldsymbol{y}_{k}\right)$ is the error function, which determines the state of element at $k$ th instant, $\eta$ is the learning rate. The ANN learns from input-to-output data. In this present consideration, radiation pattern was simulated for all 2811 possible combination of faults i.e. total number of samples for training is 2811 . We trained with all the samples since the effectiveness of the network depends on the training. Obtaining the samples took us about two days, which is a lot of time. For $\beta=2, \boldsymbol{y}_{1}$ is selected as the normal and $\boldsymbol{y}_{2}$ as the fault. Therefore, our proposed normal value selection process distinguishes the valid value(s) from the fault value(s). The regularization technique is adopted in order to avoid possible overfitting and to improve network generalization.

Training parameters determine the effectiveness of training. The training parameters and values used for the training of this network are summarized in Table I. At the end of the training, the training error and test error are depicted in Table II. As shown, even though we attempted to train the conventional or traditional direct inverse model with sufficient training iterations and hidden neurons, the training and test errors are always large because of nonuniqueness problem. We tested the trained network for the results. The outputs of the network are rounded off to determine the number of faulty elements and corresponding position. For instance, a raw output of 0.2385 , after rounding off to two significant number give the value 0.24 representing the fault in the $m_{24}$ in the entire array. The results of typical fault patterns are shown in Figure 10. In all cases considered the output of the network matches with simulation results.

This method provides good, reliable, and accurate antenna array diagnosis at $20 \mathrm{~dB}$. This is a useful feature, particularly in very harsh measurement environment. Also, our method require fewer numbers of measurements for diagnosis after training. Since the speed of diagnosis depends on the number of measurements, the proposed method offer faster diagnosis. In addition, after the successful training, which usually takes time, our system performs diagnosis in less than 5 second thereby reduced energy consumption and processing complexity. The proposed method can work at any pointing direction (beam steering), as long as the measurement or samples can be taken at the principal plane. The proposed algorithm is not direction dependent.

Although the training is time consuming, but this is not significant compared to measurement time cost required by matrix inversion [4], genetic algorithm [1], exhaustive search [3], and even CS [7]. The training is done once and for all, before installation on the base station. After the successful training, the diagnosis or fault detection is performed in much simpler and automated way.

In addition, due to the intelligence of this system, it has another advantage of performing real-time antenna array diagnosis. The availability of real-time antenna diagnosis is an asset, particularly when the replacement and manual disassembling operations are costly and consume excessive time, and in the satellite-borne installations. Therefore, recalibration of feeding network is more convenient to reinstate the radiation features via elements excitation reconfiguration. 
mANN raw output: $0.3580,0.0002,0.0001,0.0007,0.0010$

Post-processing output: $0.36,0,0,0,0$

( $\mathrm{m}_{36}$ element is faulty)

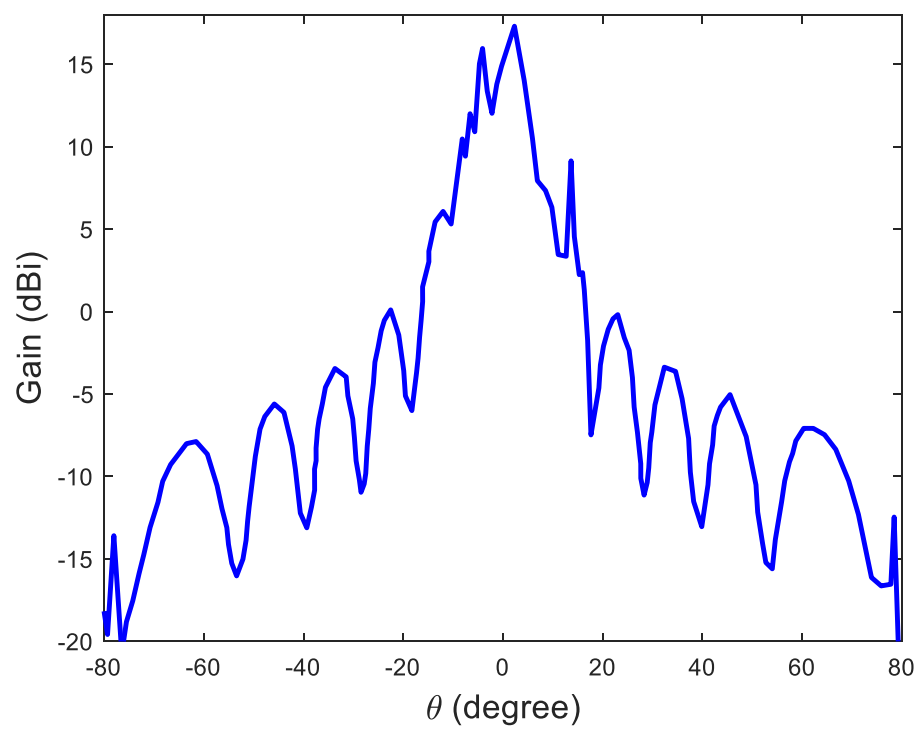

(a)

mANN raw output: $0.2780,0.8070,0.0010,0.0030,0.0030$

Post-processing output: $0.28,0.81,0,0,0$

$\left(\mathrm{m}_{28}\right.$ and $\mathrm{m}_{81}$ are faulty)

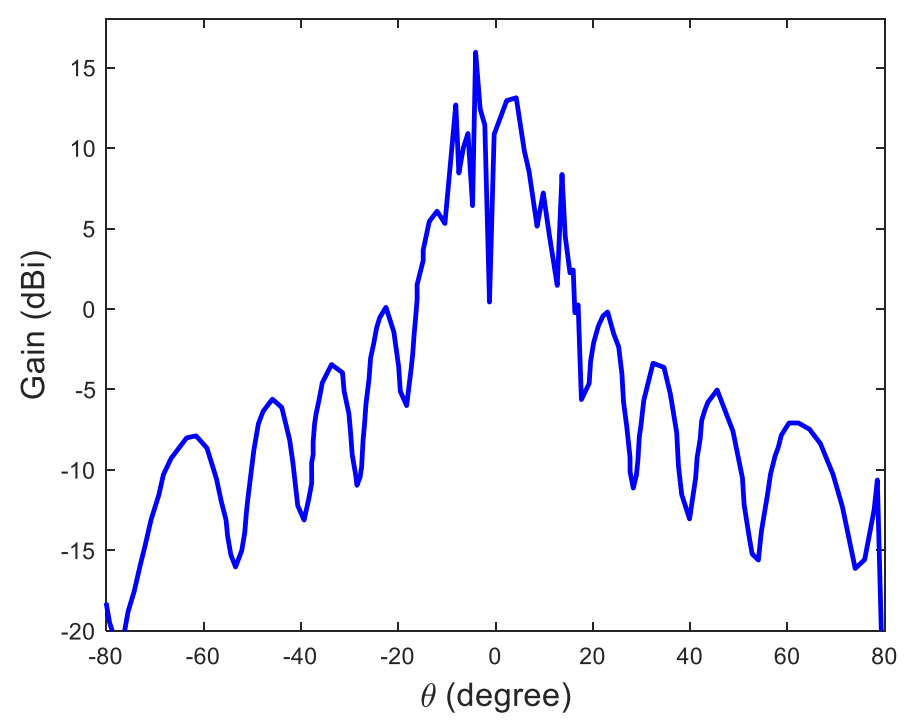

(b)

FIGURE. 10 [a, b]. Radiation pattern of fault (s) and corresponding multivalued ANN output in the insets. (a) Radiation pattern with single element $\left(m_{36}\right)$ fault (b) Radiation pattern with double elements $\left(m_{28}, m_{81}\right)$ fault. 
mANN raw output: $0.2400,0.7880,0.9690,0.0070,0.0010$

Post-processing output: $0.24,0.79,0.97,0,0$

$\left(\mathrm{m}_{24}, \mathrm{~m}_{79}\right.$, and $\mathrm{m}_{97}$ are faulty)

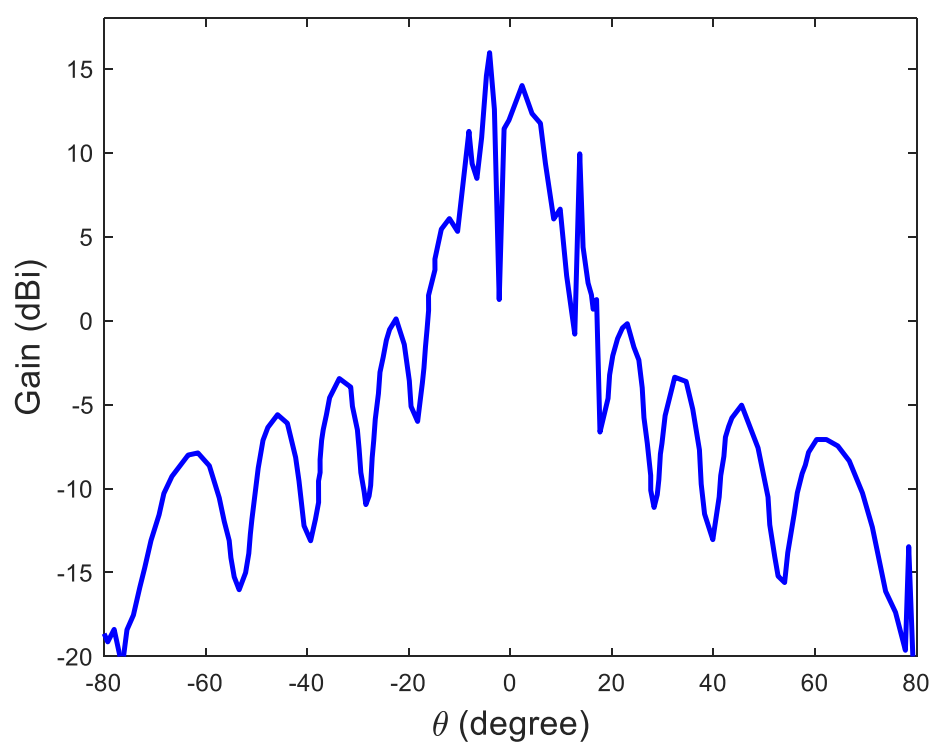

(c)

mANN raw output: $0.5280,0.8060,0.8490,0.9570,0.0030$

Post-processing output: $0.53,0.81,0.85,0.96,0$

$\left(\mathrm{m}_{53} \mathrm{~m}_{81}, \mathrm{~m}_{85}\right.$, and $\mathrm{m}_{96}$ are faulty)

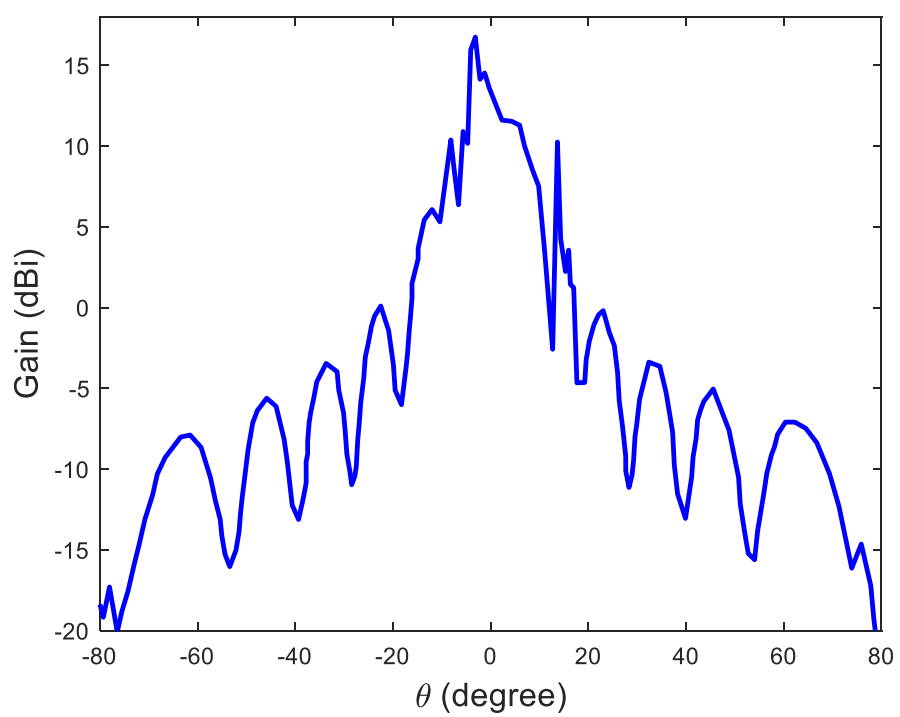

(d)

FIGURE 10 [c, d]. Radiation pattern of fault (s) and corresponding multivalued ANN output in the insets. (c) Radiation pattern with three elements

$\left(m_{24}, m_{79}, m_{97}\right)$ faults (d) Radiation pattern with four elements $\left(m_{53}, m_{81}, m_{85}, m_{96}\right)$ fault. 
mANN raw output: $0.3900,0.31000,0.7280,0.9870,0.0060$

Post-processing output: $0.39,0.310,0.73,0.99,0$

$\left(\mathrm{m}_{39}, \mathrm{~m}_{3,10}, \mathrm{~m}_{73}\right.$, and $\mathrm{m}_{99}$ are faulty)

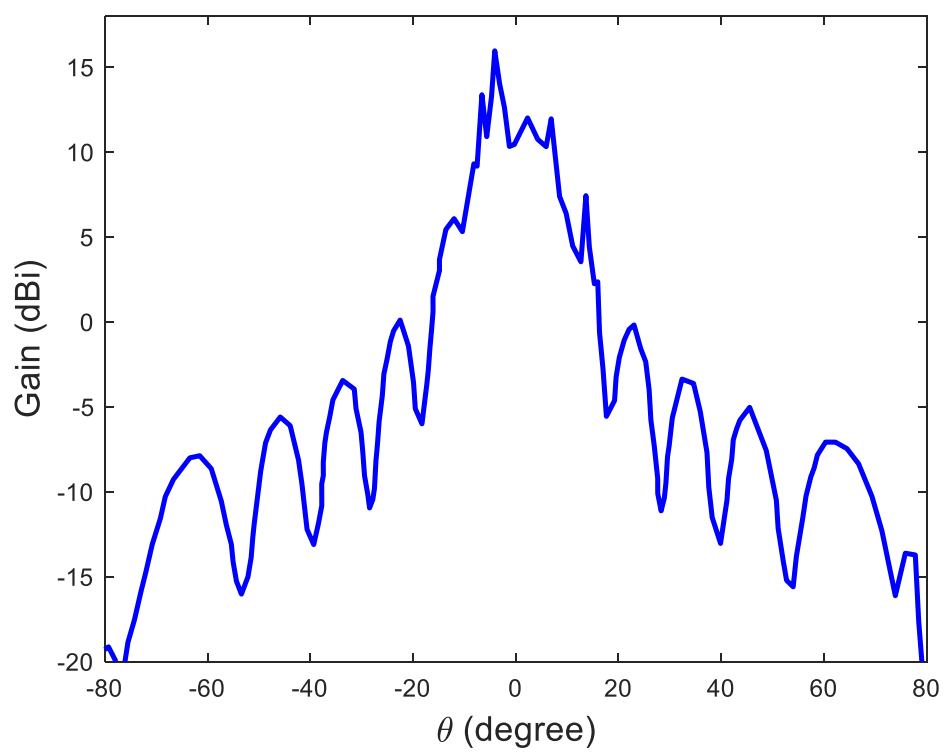

(e)

mANN raw output: $0.2580,0.4880,0.6770,0.8500,0.10100$

Post-processing output: $0.26,0.49,0.68,0.85,0.1010$

$\left(\mathrm{m}_{26}, \mathrm{~m}_{49}, \mathrm{~m}_{68}, \mathrm{~m}_{85}\right.$, and $\mathrm{m}_{10,10}$ are faulty)

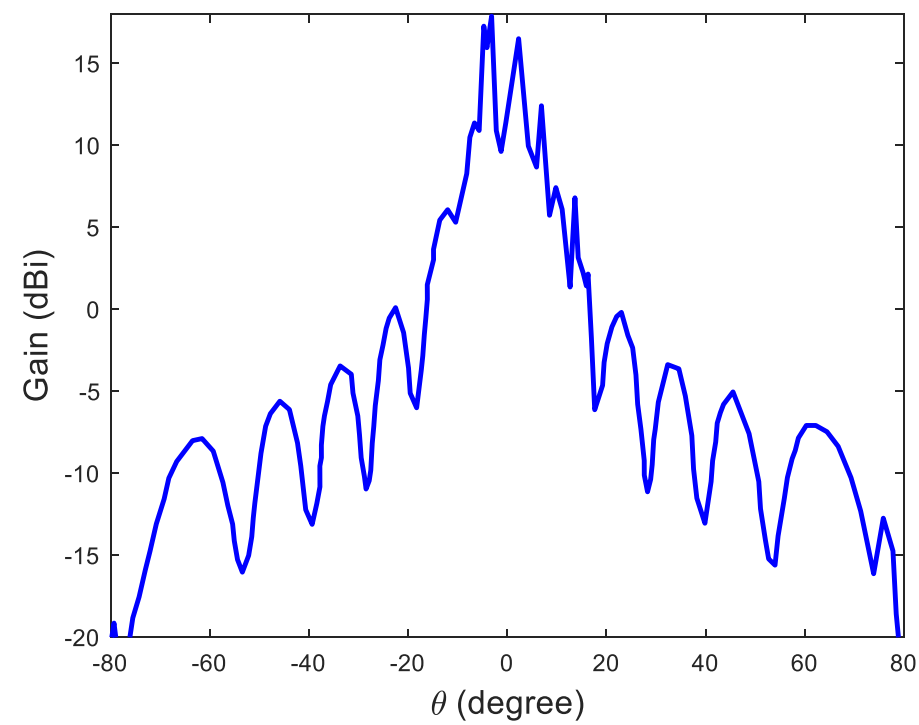

(f)

FIGURE $10[\mathrm{e}, \mathrm{f}]$. Radiation pattern of fault (s) and corresponding multivalued ANN output in the insets. (e) Radiation pattern for four elements $\left(m_{39}, m_{3,10}, m_{73}, m_{99}\right)$ faults (f) Radiation pattern for five element $\left(m_{26}, m_{49}, m_{68}, m_{85}, m_{10,10}\right)$ faults. 


\section{CONCLUSION}

In conclusion, an antenna array diagnosis method by means of multivalued inverse model has been presented. Our proposed inverse modeling method is able to solve the non-uniqueness problem, which is difficult for the direct inverse modeling method. We proposed a training procedure and test error functions towards optimal antenna array diagnosis. They are tested with various electromagnetic radiation patterns acquired from a designed antenna array, and the results obtained have good agreement with simulation results. In addition, we have applied our method to perform radiating planar structure diagnosis but can be extended to conformal antennas. The results confirm that the proposed method allows remarkable decrease in the required measurements for array diagnosis after the successful training. Although, the time taken in training the system might be long, but it takes few seconds to post-process in all cases, thereby, offers fast and accurate diagnosis with reduced complexity and lower computational burden. The proposed network finds application at the base stations in investigating the number and position of faults in antenna array, from the distorted radiation pattern caused by nonradiating elements.

ACKNOWLEDGEMENT: This research is supported in part by University Research Committee (URC) of the university of Johannesburg, South Africa.

\section{REFERENCES}

[1] J. A. Rodriguez, F. Ares, E. Moreno, and G. Franceschetti, "Genetic algorithm procedure for linear array failure correction," Electron. Lett., vol. 36, no. 3, pp. 196-198, Feb. 2000.

[2] R. Iglesias, F. Ares, M. Fernandez-Delgado, J. A. Rodriguez, J. Bregains, and S. Barro, "Element failure detection in linear antenna arrays using case-based reasoning," IEEE Antennas Propag. Mag., vol. 50, no. 4, pp. 198-204, Aug. 2008.

[3] O. M. Bucci, M. D. Migliore, G. Panariello, and P. Sgambato, "Accurate diagnosis of conformal arrays from near-field data using the matrix method," IEEE Trans. Antennas Propag., vol. 53, no. 3, pp. 1114-1120, Mar. 2005.

[4] J. A. Rodriguez-Gonzalez, F. Ares-Pena, M. Fernandez-Delgado, R. Iglesias, and S. Barro, "Rapid method for finding faulty elements in antenna arrays using far field pattern samples," IEEE Trans. Antennas Propag., vol. 57, no. 6, pp. 1679-1683, Jun. 2009.

[5] O. J. Famoriji, Z. Zhang, A. Fadamiro, Z. Khan, and F. Lin, "Active antenna array diagnosis from far-field measurements," IEEE Int. Conference on Integrated circuits, technologies and applications (ICTA), Beijing, China, Dec. 2018, pp. 1-2.S.

[6] Clauzier, S. Mikki, and Y. Antar, "Design of near-field synthesis arrays through global optimization," IEEE Trans. Antennas Propag., vol. 63, no. 1, pp. 151-165, Jan. 2015.

[7] M. D. Migliore, "A compressed sensing approach for array diagnosis from a small set of near-field measurements," IEEE Trans. Antennas Propag., vol. 59, no. 6, pp. 2127-2133, Jun. 2011.
[8] G. Oliveri, P. Rocca, and A. Massa, "Reliable diagnosis of large linear arrays-A Bayesian compressive sensing approach," IEEE Trans. Antennas Propag., vol. 60, no. 10, pp. 4627 - 4636, Oct. 2012.

[9] O. J. Famoriji, and T. Shongwe, "A recovery performance study of compressive sensing methods on antenna array diagnosis from nearfield measurements," Applied Computational Electromagnetics Society Journal, vol.36, no.8, pp. 973-980, August 2021.

[10] M. E. Eltayeb, T. Y. Al-Naffouri, and R. W. H. Jr., "Compressive sensing for millimeter wave antenna array diagnosis," IEEE Trans. Communic., vol. 66, no. 6, pp. 2708-2721, Jun. 2018.

[11] M. T. N. Kapetanakis, I. O. Vardiambasis, M. P. Ioannidou, and A. Maras, "Neural network modeling for the solution of the inverse loop antenna radiation problem," IEEE Trans. Antennas Propag., vol. 66, no. 11, pp. 6283-6290, Nov. 2018.

[12] Q.-J. Zhang and K. C. Gupta, Neural Networks for RF and Microwave Design. Norwood, MA, USA: Artech House, 2000.

[13] J. E. Rayas-Sanchez, "EM-based optimization of microwave circuits using artificial neural networks: The state-of-the-art," IEEE Trans. Microw. Theory Techn., vol. 52, no. 1, pp. 420-435, Jan. 2004.

[14] W. Na, F. Feng, C. Zhang, and Q.-J. Zhang, "A unified automated parametric modeling algorithm using knowledge-based neural network andloptimization," IEEE Trans. Microw. Theory Techn., vol. 65, no. 3,pp. 729-745, Mar. 2017.

[15] F. Feng, V.-M.-R. Gongal-Reddy, C. Zhang, J. Ma, and Q.-J. Zhang, "Parametric modeling of microwave components using adjoint neural networks and pole-residue transfer functions with EM sensitivity analysis," IEEE Trans. Microw. Theory Techn., vol. 65, no. 6, pp. 1955-1975,Jun. 2017.

[16] W. Liu, W. Na, L. Zhu, J. Ma, and Q.-J. Zhang, "A Wiener-type dynamic neural network approach to the modeling of nonlinear microwave devices," IEEE Trans. Microw. Theory Techn., vol. 65, no. 6,pp. 2043-2062, Jun. 2017.

[17] L. Zhu, Q. Zhang, K. Liu, Y. Ma, B. Peng, and S. Yan, "A novel dynamic neuro-space mapping approach for nonlinear microwave device modeling," IEEE Microw. Wireless Compon. Lett., vol. 26, no. 2, pp. 131-133, Feb. 2016.

[18] S. Koziel and A. Bekasiewicz, "Expedited geometry scaling of compact microwave passives by means of inverse surrogate modeling," IEEE Trans. Microw. Theory Techn., vol. 63, no. 12, pp. 4019-4026, Dec. 2015.

[19] H. Acikgoz, B. Jannier, Y. Le Bihan, O. Dubrunfaut, O. Meyer, and L. Pichon, "Direct and inverse modeling of a microwave sensor determining the proportion of fluids in a pipeline," IEEE Trans. Magn.,vol. 45, no. 3, pp. 1510-1513, Mar. 2009.

[20] H. Kabir, Y. Wang, M. Yu, and Q.-J. Zhang, "Neural network inverse modeling and applications to microwave filter design," IEEE Trans. Microw. Theory Techn., vol. 56, no. 4, pp. 867-879, Apr. 2008.

[21] C. Zhang, J. Jin, W Na, Q-J Zhang, and M. Yu, "Multivalued neural network inverse modeling and applications for microwave filters," IEEE Trans. Microw. Theory Techn., vol. 66, no. 8, pp. 3781-3799, Aug. 2018. 
[22] S. Selleri, S. Manetti, and G. Pelosi, "Neural network applications in microwave device design," Int. J. RF Microw. Comput.-Aided Eng., vol. 12, pp. 90-97, Jan. 2002.

[23] H. Kabir, L. Zhang, M. Yu, P. H. Aaen, J. Wood, and Q.-J. Zhang, "Smart modeling of microwave devices," IEEE Microw. Mag., vol. 11, no. 3, pp. 105-118, May 2010.

[24] H. T. Hui, "A new definition of mutual impedance for application in dipole receiving antenna arrays," IEEE Antennas Wireless Propag.Lett., vol. 3, pp. 364-367, 2004.

[25] Q. J. Zhang and K. C. Gupta, Neural Networks for RF and Microwave Design. Boston, MA, USA: Artech House, 2000.

[26] O. J. Famoriji, Z. Zhang, A. Fadamiro, Z. Zhang, R. Zakkariya, and F. Lin, "Planar array diagnostic tool for millimeter-wave wireless communication systems," Electronics, vol. 20, no. 2018, pp. 1-21, Dec. 2018.

[27] R. Mallahzadeh, and M. Taherzadeh, "Investigation of a proposed ANN-based array antenna diagnosis technique on a planar microstrip array antenna," Applied Computational Electromagnetic Society Journal, vol. 26, no. 8, pp. 667-678, Aug., 2011. 